\title{
Transition States of Vicinal Diamine-Catalyzed Aldol Reactions
}

Adam Simon, Dr. Yu-hong Lam, Prof. K. N. Houk

\section{Supporting Information}
A. Complete set of TS-1a-1h (M06-2X/def2-TZVPP//B3LYP/6-31G(d)).............S2
B. Computational results by other density functionals........................... 3

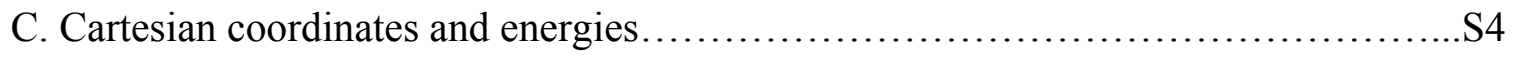




\section{A. Complete set of TS-1a-1h (M06-2X/def2-TZVPP//B3LYP/6-31G(d))}

Eight transition structures (TS-1a-1h) are shown below in Figure S1. They vary based on the conformation of the cyclic transition state. Their conformations and free energies of activation are listed in Table S1.

Table S1. Intermolecular Aldol Addition Transition Structures

\begin{tabular}{|c|c|c|c|}
\hline Entry & TS & Ring Conformation & $\Delta \Delta G^{\ddagger}$ \\
\hline 1 & TS-1a & crown (chair-chair) & 0 \\
\hline 2 & TS-1b & chair-boat & 0.6 \\
\hline 3 & TS-1c & boat-chair & 2.6 \\
\hline 4 & TS-1d & boat-boat & 3.3 \\
\hline 5 & TS-1e & twist-boat-chair & 3.5 \\
\hline 6 & TS-1f & twist-crown & 5.2 \\
\hline 7 & TS-1g & twist-chair-boat & 5.8 \\
\hline 8 & TS-1h & twist-boat-boat & 6.1 \\
\hline
\end{tabular}

M06-2X/def2-TZVPP//B3LYP/6-31G(d). The relative free energies of activation $\left(\Delta \Delta G^{\ddagger}\right)$ are reported in $\mathrm{kcal} / \mathrm{mol}$ relative to the lowest-energy transition structure, TS-1a.
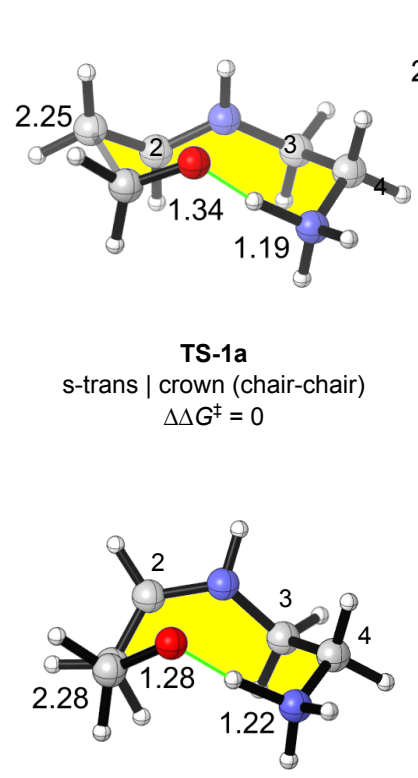

TS-1c s-cis | boat-chair $\Delta \Delta G^{\ddagger}=2.6$

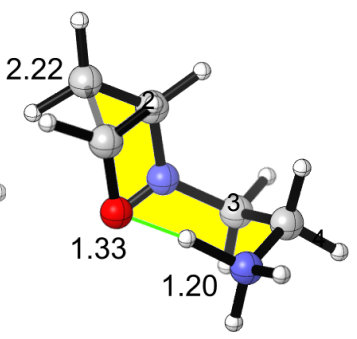

TS-1b

s-trans | chair-boat $\Delta \Delta G^{\ddagger}=0.6$

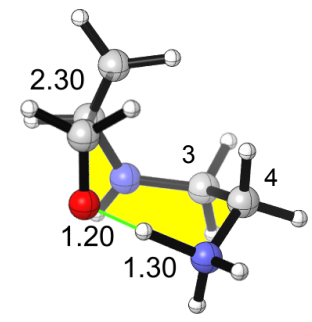

TS-1d s-cis | boat-boat $\Delta \Delta G^{\ddagger}=3.3$

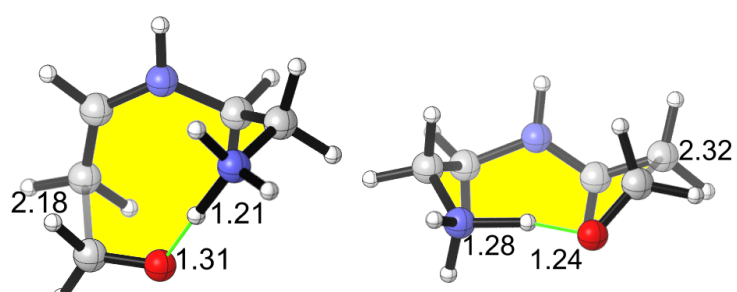

TS-1f s-trans | twist-crown $\Delta \Delta G^{\ddagger}=5.2$

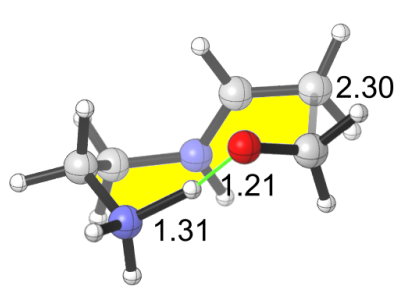

TS-1g s-trans | twist-chair-boat $\Delta \Delta G^{\ddagger}=5.8$

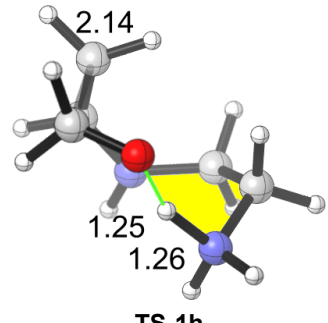

s-cis | twist-boat-boat $\Delta \Delta G^{\ddagger}=6.1$

Figure S1. Transition structures for the aldol reaction of formaldehyde and the enamine of ethylenediamine and acetaldehyde (M06-2X/def2-TZVPP//B3LYP/6-31G(d)). The relative free energies of activation compared with TS-1a are reported in $\mathrm{kcal} / \mathrm{mol}$. The hydrogen-bonded nine-membered rings are color-filled. 


\section{B. Computational results with other density functionals}

In addition to the M06-2X/def2-TZVPP//B3LYP/6-31G(d) results discussed in the Main Text, transition structures were also fully optimized at the B3LYP/6-31G(d), B3LYP/def2-TZVPP, $\omega$ B97X-D/def2-TZVPP, and M06-2X/def2-TZVPP level of theory. The results are summarized in Table S2. B3LYP geometries and energies are consistent with other levels of theory and basis sets. M06-2X predicts smaller differences in energy between conformations. The trend of conformational preferences remains the same despite the level of theory except where the twist-boat-chair becomes higher in energy than the boat-boat with M062-2X/def2-TZVPP. The combination of B3LYP geometries and M06-2X single point energies yielded reasonable balances between accuracy and computational efficiency for larger systems.

Table S2. Selectivity Data $\left(\Delta \Delta G^{\sharp}\right)$ Predicted for Intermolecular Aldol Additions of Formaldehyde and the Enamine of Ethylenediamine and Acetaldehyde.

\begin{tabular}{|c|c|c|c|c|c|}
\hline Conformation & $\begin{array}{c}\text { B3LYP / } \\
6-31 G(d)\end{array}$ & $\begin{array}{c}\text { B3LYP / } \\
\text { def2-TZVPP }\end{array}$ & $\begin{array}{c}\omega \text { B97X-D / } \\
\text { def2-TZVPP }\end{array}$ & $\begin{array}{c}\text { M06-2X / } \\
\text { def2-TZVPP }\end{array}$ & $\begin{array}{c}\text { M06-2X/def2-TZVPP } \\
/ / \text { B3LYP/6-31G(d) }\end{array}$ \\
\hline crown & 0 & 0 & 0 & 0 & 0 \\
\hline chair-boat & 0.9 & 1.0 & 0.8 & 0.4 & 0.6 \\
\hline boat-chair & 2.3 & 2.0 & 1.8 & 2.1 & 2.2 \\
\hline boat-boat & 4.6 & 4.6 & 3.4 & 2.9 & 3.3 \\
\hline t-boat-chair & 3.3 & 3.3 & 3.1 & 2.7 & 3.5 \\
\hline t-crown & 4.5 & 4.6 & 4.7 & 4.7 & 5.2 \\
\hline t-chair-boat & 5.9 & 6.1 & 5.8 & N/A & 5.8 \\
\hline t-boat-boat & 6.5 & 6.6 & 6.1 & N/A & 6.1 \\
\hline
\end{tabular}




\section{Cartesian coordinates and energies}

$\begin{array}{lrrr}\text { TS-1a } & & & \\ \mathrm{C} & -0.96798 & -1.25179 & 0.40651 \\ \mathrm{C} & -2.24539 & -0.92832 & 0.02857 \\ \mathrm{H} & -2.50847 & -0.86492 & -1.02357 \\ \mathrm{~N} & 0.10588 & -1.17426 & -0.40551 \\ \mathrm{C} & 1.49311 & -1.21977 & 0.02504 \\ \mathrm{C} & 2.23122 & 0.10165 & -0.22867 \\ \mathrm{H} & 1.51183 & -1.47688 & 1.08971 \\ \mathrm{H} & 2.23823 & 0.33986 & -1.29605 \\ \mathrm{~N} & 1.56063 & 1.25289 & 0.45900 \\ \mathrm{H} & -0.06850 & -1.02133 & -1.39283 \\ \mathrm{H} & 0.44108 & 1.37760 & 0.07686 \\ \mathrm{H} & -3.05964 & -1.10358 & 0.72298 \\ \mathrm{C} & -1.79994 & 1.27594 & 0.14165 \\ \mathrm{O} & -0.74954 & 1.49565 & -0.52697 \\ \mathrm{H} & 2.03617 & -2.01227 & -0.50337 \\ \mathrm{H} & -2.76890 & 1.43666 & -0.33740 \\ \mathrm{H} & -1.79663 & 1.31185 & 1.23780 \\ \mathrm{H} & -0.74048 & -1.47703 & 1.44717 \\ \mathrm{H} & 2.04937 & 2.12800 & 0.24816 \\ \mathrm{H} & 1.58491 & 1.14443 & 1.47676 \\ \mathrm{H} & 3.26848 & 0.02314 & 0.11020\end{array}$

Electronic energy $=-382.80785$

Zero-point electronic energy $=-382.62044$

Enthalpy $=-382.610578$

Free energy $=-382.653615$

Free energy with quasiharmonic approximation $=-382.653465$

Frequencies $=-386.517085 .1148157 .0255-386.516385 .0838157 .0245$

SCF $($ M06-2X/def2-TZVPP $)=-382.7843088$

TS- $1 b$

$\begin{array}{lrrc}\mathrm{C} & 0.90337 & -1.27551 & 0.32996 \\ \mathrm{C} & 2.20342 & -0.88864 & 0.11560 \\ \mathrm{H} & 2.56743 & -0.73613 & -0.89655 \\ \mathrm{~N} & -0.08277 & -1.13405 & -0.57680 \\ \mathrm{C} & -1.50549 & -1.20320 & -0.26852 \\ \mathrm{C} & -2.00697 & -0.03606 & 0.59688 \\ \mathrm{H} & -2.04914 & -1.24072 & -1.21726 \\ \mathrm{H} & -1.54341 & -0.06339 & 1.58688 \\ \mathrm{~N} & -1.65464 & 1.29086 & -0.00246 \\ \mathrm{H} & 0.18362 & -0.82485 & -1.50363 \\ \mathrm{H} & -0.47647 & 1.41127 & -0.17938 \\ \mathrm{H} & 2.95021 & -1.11904 & 0.86775 \\ \mathrm{C} & 1.71844 & 1.26463 & 0.30494\end{array}$




$\begin{array}{lrrr}\mathrm{O} & 0.80023 & 1.52003 & -0.53092 \\ \mathrm{H} & -1.73279 & -2.13545 & 0.26083 \\ \mathrm{H} & 2.75317 & 1.46975 & 0.01963 \\ \mathrm{H} & -3.09081 & -0.10801 & 0.72863 \\ \mathrm{H} & -1.97909 & 2.05434 & 0.59758 \\ \mathrm{H} & -2.11326 & 1.42383 & -0.90917 \\ \mathrm{H} & 0.58265 & -1.61429 & 1.31396 \\ \mathrm{H} & 1.51799 & 1.25085 & 1.38316\end{array}$

Electronic energy $=-382.806279$

Zero-point electronic energy $=-382.619052$

Enthalpy $=-382.609153$

Free energy $=-382.652212$

Free energy with quasiharmonic approximation $=-382.652056$

Frequencies $=-459.929284 .5457152 .0744-459.928884 .5184152 .0723$

SCF $($ M06-2X/def2-TZVPP $)=-382.7831948$

TS-1c

$\begin{array}{lrrr}\mathrm{C} & -1.23043 & -1.29892 & -0.24307 \\ \mathrm{C} & -1.76636 & -0.73303 & 0.88460 \\ \mathrm{H} & -1.15990 & -0.49918 & 1.75421 \\ \mathrm{~N} & 0.07175 & -1.35077 & -0.59655 \\ \mathrm{C} & 1.22777 & -1.19917 & 0.28077 \\ \mathrm{C} & 2.14152 & -0.01368 & -0.06336 \\ \mathrm{H} & 0.88309 & -1.14198 & 1.31705 \\ \mathrm{H} & 2.32158 & 0.02814 & -1.14081 \\ \mathrm{~N} & 1.56326 & 1.30979 & 0.33377 \\ \mathrm{H} & 0.26871 & -1.74133 & -1.50946 \\ \mathrm{H} & 0.44833 & 1.46771 & -0.13642 \\ \mathrm{H} & -2.82624 & -0.86601 & 1.07047 \\ \mathrm{C} & -1.72184 & 1.35227 & -0.03456 \\ \mathrm{O} & -0.68373 & 1.49526 & -0.74110 \\ \mathrm{H} & 1.83942 & -2.10627 & 0.20976 \\ \mathrm{H} & -1.90034 & -1.64187 & -1.02801 \\ \mathrm{H} & -2.68295 & 1.24383 & -0.54180 \\ \mathrm{H} & -1.75856 & 1.70403 & 1.00294 \\ \mathrm{H} & 2.15286 & 2.07252 & -0.01103 \\ \mathrm{H} & 1.53512 & 1.40506 & 1.35224 \\ \mathrm{H} & 3.10575 & -0.14591 & 0.43679\end{array}$

Electronic energy $=-382.803588$

Zero-point electronic energy $=-382.616581$

Enthalpy $=-382.606558$

Free energy $=-382.650145$

Free energy with quasiharmonic approximation $=-382.64982$

Frequencies $=-564.566070 .6763130 .3277-564.56570 .549130 .2877$

SCF $(\mathrm{M} 06-2 \mathrm{X} / \mathrm{def} 2-\mathrm{TZVPP})=-382.7801266$ 


$\begin{array}{lrrc}\text { TS-1d } & & & \\ \mathrm{C} & 1.12480 & -1.27794 & -0.34337 \\ \mathrm{C} & 1.57846 & -0.91004 & 0.89695 \\ \mathrm{H} & 0.90711 & -0.79896 & 1.74241 \\ \mathrm{~N} & -0.14675 & -1.23049 & -0.79024 \\ \mathrm{C} & -1.36242 & -1.21053 & 0.02673 \\ \mathrm{C} & -1.70365 & 0.10839 & 0.74427 \\ \mathrm{H} & -2.18540 & -1.47700 & -0.64288 \\ \mathrm{H} & -1.04479 & 0.28013 & 1.59822 \\ \mathrm{~N} & -1.58247 & 1.30519 & -0.13592 \\ \mathrm{H} & -0.28096 & -1.48240 & -1.76019 \\ \mathrm{H} & -0.33119 & 1.50130 & -0.42630 \\ \mathrm{H} & 2.62012 & -1.09021 & 1.13802 \\ \mathrm{C} & 1.66259 & 1.28723 & 0.21082 \\ \mathrm{O} & 0.83445 & 1.52128 & -0.72178 \\ \mathrm{H} & -1.30963 & -1.99336 & 0.79359 \\ \mathrm{H} & 2.71916 & 1.23007 & -0.05200 \\ \mathrm{H} & 1.42430 & 1.52371 & 1.25215 \\ \mathrm{H} & -2.72347 & 0.01840 & 1.13267 \\ \mathrm{H} & -1.96492 & 2.12982 & 0.33242 \\ \mathrm{H} & -2.11591 & 1.19139 & -1.00154 \\ \mathrm{H} & 1.84832 & -1.50402 & -1.12332 \\ \mathrm{El} & -1.59\end{array}$

Electronic energy $=-382.800008$

Zero-point electronic energy $=-382.613037$

Enthalpy $=-382.603053$

Free energy $=-382.64616$

Free energy with quasiharmonic approximation $=-382.646157$

Frequencies $=-723.44999 .7400 \quad 149.3802 \quad-723.4489 \quad 99.6641149 .3515$

SCF $(\mathrm{M} 06-2 \mathrm{X} / \mathrm{def} 2-\mathrm{TZVPP})=-382.778533907$

TS-1e

$\begin{array}{lrrr}\mathrm{C} & 1.64010 & -1.38878 & -0.17661 \\ \mathrm{O} & 0.56497 & -1.73521 & 0.39871 \\ \mathrm{H} & 2.58739 & -1.65539 & 0.29914 \\ \mathrm{H} & 1.67907 & -1.24570 & -1.26333 \\ \mathrm{~N} & -0.18452 & 1.46013 & -0.38646 \\ \mathrm{H} & -0.62021 & 2.10012 & -1.04029 \\ \mathrm{C} & -1.01395 & 1.01298 & 0.72920 \\ \mathrm{H} & -0.38016 & 0.48066 & 1.43820 \\ \mathrm{H} & -1.45262 & 1.87247 & 1.25050 \\ \mathrm{C} & -2.15793 & 0.09579 & 0.27216 \\ \mathrm{H} & -2.65155 & -0.32052 & 1.15365 \\ \mathrm{H} & -2.90821 & 0.64422 & -0.30572 \\ \mathrm{H} & -0.57867 & -1.43507 & -0.15791 \\ \mathrm{C} & 1.16355 & 1.34938 & -0.47725 \\ \mathrm{C} & 2.02498 & 0.68375 & 0.36520\end{array}$




$\begin{array}{llll}\mathrm{H} & 1.55904 & 1.79120 & -1.38992 \\ \mathrm{H} & 3.08479 & 0.80185 & 0.16505 \\ \mathrm{H} & 1.76264 & 0.46266 & 1.39367 \\ \mathrm{H} & -1.53367 & -0.76592 & -1.52982 \\ \mathrm{~N} & -1.64951 & -1.04914 & -0.55460 \\ \mathrm{H} & -2.31480 & -1.82634 & -0.54922\end{array}$

Electronic energy $=-382.802606$

Zero-point electronic energy $=-382.614951$

Enthalpy $=-382.605034$

Free energy $=-382.648317$

Free energy with quasiharmonic approximation $=-382.648275$

Frequencies $=-593.193295 .6467116 .1276-593.192295 .5512116 .1224$

SCF $($ M06-2X/def2-TZVPP $)=-382.7786928$

$\begin{array}{lrrr}\text { TS-1f } & & & \\ \mathrm{C} & -1.76770 & -1.19713 & -0.40526 \\ \mathrm{O} & -0.91983 & -1.73695 & 0.36344 \\ \mathrm{H} & -2.82336 & -1.41084 & -0.23152 \\ \mathrm{H} & -1.48018 & -0.86421 & -1.40827 \\ \mathrm{~N} & 0.17867 & 1.43771 & -0.27625 \\ \mathrm{H} & 0.04227 & 1.75041 & -1.23157 \\ \mathrm{C} & 1.54830 & 1.20533 & 0.15563 \\ \mathrm{H} & 2.17822 & 2.03209 & -0.18864 \\ \mathrm{H} & 1.57332 & 1.22442 & 1.25118 \\ \mathrm{C} & 2.15947 & -0.10090 & -0.37752 \\ \mathrm{H} & 2.02211 & -0.16939 & -1.46052 \\ \mathrm{H} & 3.23593 & -0.10032 & -0.17663 \\ \mathrm{H} & 0.28035 & -1.47717 & 0.18757 \\ \mathrm{C} & -0.91813 & 1.17717 & 0.46638 \\ \mathrm{C} & -2.20090 & 1.04205 & 0.00844 \\ \mathrm{H} & -0.70496 & 0.95416 & 1.51065 \\ \mathrm{H} & -3.00568 & 0.98771 & 0.73228 \\ \mathrm{H} & -2.47253 & 1.32012 & -1.00700 \\ \mathrm{H} & 1.79394 & -1.39336 & 1.21833 \\ \mathrm{~N} & 1.54767 & -1.32604 & 0.22614 \\ \mathrm{H} & 1.94671 & -2.16065 & -0.21256\end{array}$

Electronic energy $=-382.798975$

Zero-point electronic energy $=-382.612852$

Enthalpy $=-382.602592$

Free energy $=-382.646713$

Free energy with quasiharmonic approximation $=-382.646284$

Frequencies $=-865.834163 .2310122 .5372-865.833863 .1096122 .4884$

SCF $($ M06-2X/def2-TZVPP $)=-382.7743692$

TS-1g

$\begin{array}{llll}\text { C } & 3.60616 & -1.13452 & 0.75464\end{array}$ 


$\begin{array}{lrrc}\mathrm{H} & 3.95743 & -1.71081 & 1.61927 \\ \mathrm{~N} & 2.35902 & -1.69013 & 0.23163 \\ \mathrm{~N} & 2.87797 & 1.20232 & 0.13520 \\ \mathrm{C} & -0.29765 & 0.13065 & -0.17939 \\ \mathrm{O} & 0.41180 & 0.96208 & 0.46518 \\ \mathrm{H} & 0.00439 & -0.19614 & -1.17890 \\ \mathrm{C} & 1.21081 & -1.80231 & 0.92934 \\ \mathrm{C} & -0.02575 & -2.08070 & 0.40681 \\ \mathrm{H} & -0.84626 & -2.26251 & 1.09114 \\ \mathrm{H} & -0.13477 & -2.47602 & -0.60028 \\ \mathrm{C} & 3.51398 & 0.34044 & 1.17711 \\ \mathrm{H} & 2.90753 & 0.45150 & 2.08083 \\ \mathrm{H} & 4.52255 & 0.69787 & 1.40889 \\ \mathrm{H} & 1.57758 & 1.05381 & 0.17290 \\ \mathrm{H} & 2.37494 & -2.05804 & -0.71195 \\ \mathrm{H} & 4.35937 & -1.25496 & -0.02958 \\ \mathrm{H} & 3.28634 & 1.04055 & -0.78813 \\ \mathrm{H} & 3.04605 & 2.18769 & 0.35406 \\ \mathrm{H} & -1.35600 & 0.06586 & 0.07405 \\ \mathrm{H} & 1.28913 & -1.51436 & 1.97636\end{array}$

Electronic energy $=-382.79695$

Zero-point electronic energy $=-382.610669$

Enthalpy $=-382.600391$

Free energy $=-382.64449$

Free energy with quasiharmonic approximation $=-382.644133$

Frequencies $=-828.666068 .2176127 .8176-828.665468 .1774127 .8108$

SCF (M06-2X/def2-TZVPP) $=-382.773436399$

$\begin{array}{lrrr}\text { TS-1h } & & & \\ \mathrm{C} & -1.83390 & -1.17288 & -0.17469 \\ \mathrm{O} & -0.78460 & -1.81976 & 0.14508 \\ \mathrm{H} & -2.73658 & -1.36403 & 0.41136 \\ \mathrm{H} & -2.00347 & -0.86239 & -1.21100 \\ \mathrm{~N} & 0.31088 & 1.42388 & -0.62300 \\ \mathrm{H} & 0.57391 & 1.90996 & -1.47024 \\ \mathrm{C} & 1.40550 & 1.20355 & 0.32756 \\ \mathrm{H} & 2.28717 & 1.66757 & -0.12229 \\ \mathrm{H} & 1.20106 & 1.73862 & 1.26236 \\ \mathrm{C} & 1.72579 & -0.26433 & 0.67978 \\ \mathrm{H} & 2.76068 & -0.31195 & 1.03048 \\ \mathrm{H} & 1.08090 & -0.63011 & 1.48183 \\ \mathrm{H} & 1.78378 & -0.79639 & -1.36380 \\ \mathrm{C} & -1.01434 & 1.38778 & -0.36976 \\ \mathrm{C} & -1.66764 & 0.78154 & 0.68270 \\ \mathrm{H} & -1.61188 & 1.81830 & -1.17130 \\ \mathrm{H} & -2.71459 & 1.03330 & 0.81752\end{array}$




$\begin{array}{rrrr}\mathrm{H} & -1.13866 & 0.46608 & 1.57623 \\ \mathrm{H} & 0.30978 & -1.54093 & -0.37913 \\ \mathrm{~N} & 1.51770 & -1.20324 & -0.46533 \\ \mathrm{H} & 2.07533 & -2.05130 & -0.33907\end{array}$

Electronic energy $=-382.796926$

Zero-point electronic energy $=-\mathbf{3 8 2 . 6 0 9 6 0 8}$

Enthalpy $=-382.599528$

Free energy $=-382.64321$

Free energy with quasiharmonic approximation $=-382.64314$

Frequencies $=-886.088992 .8283102 .9872-886.088792 .7525102 .91$

SCF $($ M06-2X/def2-TZVPP $)=-382.7739884$

TS-2a

$\begin{array}{lrrr}\mathrm{C} & 3.44537 & -1.29378 & 0.09547 \\ \mathrm{H} & 3.88671 & -1.16181 & 1.08282 \\ \mathrm{~N} & 2.01446 & -1.63681 & 0.21379 \\ \mathrm{C} & 1.26116 & -1.51455 & 1.30466 \\ \mathrm{C} & -0.15018 & -1.54211 & 1.19083 \\ \mathrm{H} & -0.58520 & -2.04934 & 0.33609 \\ \mathrm{H} & -0.70043 & -1.69219 & 2.11531 \\ \mathrm{C} & 3.64150 & -0.02895 & -0.76948 \\ \mathrm{H} & 3.30900 & -0.22824 & -1.79063 \\ \mathrm{C} & 3.44728 & 1.88759 & 0.91511 \\ \mathrm{C} & 2.81624 & 2.23855 & -1.41845 \\ \mathrm{H} & 2.72955 & 1.74282 & 1.72314 \\ \mathrm{H} & 4.39461 & 1.42400 & 1.19297 \\ \mathrm{C} & 3.58126 & 3.37167 & 0.53443 \\ \mathrm{C} & 2.61845 & 3.53897 & -0.65126 \\ \mathrm{H} & 2.00088 & 1.96200 & -2.08787 \\ \mathrm{H} & 3.76320 & 2.23327 & -1.96525 \\ \mathrm{H} & 3.34135 & 4.02184 & 1.37883 \\ \mathrm{H} & 4.60701 & 3.58966 & 0.22054 \\ \mathrm{H} & 1.58097 & 3.61777 & -0.30806 \\ \mathrm{~N} & 2.89555 & 1.18817 & -0.32364 \\ \mathrm{H} & 1.85684 & 0.91323 & -0.15889 \\ \mathrm{C} & -2.85981 & 0.26472 & 1.44986 \\ \mathrm{C} & -1.92939 & 0.23240 & 0.40040 \\ \mathrm{C} & -2.38650 & 0.16974 & -0.92354 \\ \mathrm{C} & -3.74739 & 0.12544 & -1.20214 \\ \mathrm{C} & -4.65076 & 0.15123 & -0.13728 \\ \mathrm{C} & -4.22502 & 0.22388 & 1.19072 \\ \mathrm{H} & -1.65840 & 0.16998 & -1.72709 \\ \mathrm{H} & -4.11467 & 0.07752 & -2.21971 \\ \mathrm{H} & -4.95344 & 0.25147 & 1.99127 \\ \mathrm{C} & -0.44445 & 0.30165 & 0.67991 \\ \mathrm{O} & 0.32793 & 0.63398 & -0.30230\end{array}$




$\begin{array}{lrrr}\mathrm{H} & -2.51326 & 0.32854 & 2.47800 \\ \mathrm{H} & -0.24877 & 0.74785 & 1.67108 \\ \mathrm{H} & 4.70613 & 0.21740 & -0.80433 \\ \mathrm{H} & 2.84545 & 4.41162 & -1.26816 \\ \mathrm{~N} & -6.08434 & 0.10859 & -0.42077 \\ \mathrm{O} & -6.86892 & 0.13828 & 0.53164 \\ \mathrm{O} & -6.44461 & 0.04350 & -1.59950 \\ \mathrm{C} & 1.57866 & -2.36806 & -1.01052 \\ \mathrm{H} & 0.92496 & -3.18621 & -0.70251 \\ \mathrm{H} & 1.01402 & -1.70011 & -1.66274 \\ \mathrm{C} & 4.01125 & -2.51309 & -0.65280 \\ \mathrm{H} & 4.96549 & -2.29847 & -1.14115 \\ \mathrm{H} & 4.17333 & -3.32241 & 0.06488 \\ \mathrm{C} & 2.89141 & -2.87747 & -1.65420 \\ \mathrm{H} & 3.05591 & -2.39583 & -2.62166 \\ \mathrm{H} & 2.84989 & -3.95400 & -1.83289 \\ \mathrm{C} & 1.88879 & -1.14892 & 2.62398 \\ \mathrm{H} & 2.67376 & -1.86480 & 2.89053 \\ \mathrm{H} & 2.34170 & -0.15229 & 2.61842 \\ \mathrm{H} & 1.13130 & -1.16248 & 3.40844\end{array}$

Electronic energy $=-1130.540062$

Zero-point electronic energy $=-1130.078817$

Enthalpy $=-1130.054782$

Free energy $=-1130.132596$

Free energy with quasiharmonic approximation $=-1130.127972$

Frequencies $=-257.380127 .221931 .3808-257.378526 .777631 .309$

SCF $($ M06-2X/def2-TZVPP $)=-1130.486978$

$\begin{array}{lrrr}\text { TS-2b } & & & \\ \mathrm{C} & 3.20579 & -0.37952 & 1.08495 \\ \mathrm{H} & 2.76766 & -0.19876 & 2.06608 \\ \mathrm{~N} & 2.41900 & -1.38746 & 0.34582 \\ \mathrm{C} & 1.19840 & -1.81529 & 0.64392 \\ \mathrm{C} & 0.45527 & -2.53707 & -0.32962 \\ \mathrm{H} & 1.02290 & -3.09923 & -1.06408 \\ \mathrm{H} & -0.39777 & -3.08703 & 0.05503 \\ \mathrm{C} & 3.31452 & 0.92972 & 0.27483 \\ \mathrm{H} & 3.85023 & 0.74140 & -0.65731 \\ \mathrm{C} & 1.28967 & 2.28297 & 1.02252 \\ \mathrm{C} & 2.20902 & 2.58487 & -1.23183 \\ \mathrm{H} & 0.31224 & 1.81146 & 1.13488 \\ \mathrm{H} & 1.85282 & 2.15386 & 1.94730 \\ \mathrm{C} & 1.16331 & 3.74548 & 0.57600 \\ \mathrm{C} & 1.16143 & 3.65742 & -0.95728 \\ \mathrm{H} & 2.10301 & 2.05602 & -2.17931 \\ \mathrm{H} & 3.22102 & 2.98747 & -1.14557\end{array}$




$\begin{array}{lccc}\mathrm{H} & 0.26107 & 4.20749 & 0.98347 \\ \mathrm{H} & 2.02605 & 4.32389 & 0.92233 \\ \mathrm{H} & 0.18081 & 3.34605 & -1.33401 \\ \mathrm{~N} & 2.01646 & 1.56534 & -0.11280 \\ \mathrm{H} & 1.38380 & 0.80760 & -0.54502 \\ \mathrm{C} & -0.19950 & -1.13520 & -1.44512 \\ \mathrm{O} & 0.66556 & -0.18048 & -1.58685 \\ \mathrm{H} & 3.88779 & 1.66043 & 0.85129 \\ \mathrm{H} & 1.42379 & 4.60216 & -1.43960 \\ \mathrm{C} & 3.28960 & -2.02289 & -0.68539 \\ \mathrm{H} & 3.07375 & -3.09205 & -0.70428 \\ \mathrm{H} & 3.05981 & -1.60355 & -1.66661 \\ \mathrm{C} & 4.59214 & -1.04162 & 1.15848 \\ \mathrm{H} & 5.38910 & -0.32121 & 1.36027 \\ \mathrm{H} & 4.58782 & -1.77759 & 1.96744 \\ \mathrm{C} & 4.73635 & -1.73151 & -0.21745 \\ \mathrm{H} & 5.24868 & -1.08322 & -0.93313 \\ \mathrm{H} & 5.31767 & -2.65286 & -0.14340 \\ \mathrm{C} & 0.52684 & -1.36772 & 1.91330 \\ \mathrm{H} & -0.42773 & -1.88166 & 2.02765 \\ \mathrm{H} & 1.14911 & -1.59620 & 2.78509 \\ \mathrm{H} & 0.32563 & -0.29129 & 1.92409 \\ \mathrm{H} & -0.30917 & -1.81036 & -2.31314 \\ \mathrm{C} & -1.57951 & -0.80941 & -0.89205 \\ \mathrm{C} & -2.58806 & -1.78713 & -0.83374 \\ \mathrm{C} & -1.89505 & 0.50398 & -0.52224 \\ \mathrm{C} & -3.86350 & -1.47584 & -0.38165 \\ \mathrm{H} & -2.37656 & -2.80427 & -1.15199 \\ \mathrm{C} & -3.16565 & 0.83894 & -0.06331 \\ \mathrm{H} & -1.13987 & 1.27269 & -0.63087 \\ \mathrm{C} & -4.13408 & -0.16120 & 0.00803 \\ \mathrm{H} & -4.64299 & -2.22563 & -0.33181 \\ \mathrm{H} & -3.41307 & 1.85308 & 0.22457 \\ \mathrm{~N} & -5.47261 & 0.17702 & 0.48862 \\ \mathrm{O} & -5.69542 & 1.34424 & 0.82207 \\ \mathrm{O} & -6.31756 & -0.72089 & 0.54041 \\ \mathrm{E} & -.359\end{array}$

Electronic energy $=-1130.534732$

Zero-point electronic energy $=-1130.072843$

Enthalpy $=-1130.049001$

Free energy $=-1130.125805$

Free energy with quasiharmonic approximation $=-1130.121754$

Frequencies $=-238.080828 .627131 .7840-238.079928 .443530 .2307$

SCF (M06-2X/def2-TZVPP) $=-1130.48456967$

TS-2c

$\begin{array}{llll}\text { C } & 3.53550 & -1.32352 & 0.06649\end{array}$ 


\begin{tabular}{|c|c|c|c|}
\hline $\mathrm{H}$ & 4.02460 & -2.05890 & 0.71478 \\
\hline $\mathrm{N}$ & 2.09173 & -1.67333 & -0.05124 \\
\hline $\mathrm{N}$ & 2.93175 & 1.18623 & 0.28015 \\
\hline $\mathrm{C}$ & -2.84804 & -0.11787 & 1.45619 \\
\hline $\mathrm{C}$ & -1.90101 & 0.09810 & 0.44381 \\
\hline $\mathrm{C}$ & -2.33577 & 0.34700 & -0.86616 \\
\hline $\mathrm{C}$ & -3.69147 & 0.36746 & -1.17002 \\
\hline $\mathrm{C}$ & -4.61184 & 0.14261 & -0.14371 \\
\hline $\mathrm{C}$ & -4.20849 & -0.09793 & 1.17142 \\
\hline $\mathrm{H}$ & -1.59506 & 0.53390 & -1.63585 \\
\hline $\mathrm{H}$ & -4.04237 & 0.55800 & -2.17652 \\
\hline $\mathrm{H}$ & -4.95017 & -0.25957 & 1.94339 \\
\hline $\mathrm{C}$ & -0.42711 & 0.10417 & 0.75963 \\
\hline $\mathrm{O}$ & 0.36939 & 0.65476 & -0.08509 \\
\hline $\mathrm{H}$ & -2.51763 & -0.29834 & 2.47577 \\
\hline $\mathrm{H}$ & -0.23898 & 0.24766 & 1.83783 \\
\hline $\mathrm{C}$ & 4.08613 & -1.48373 & -1.37007 \\
\hline $\mathrm{H}$ & 5.09936 & -1.89169 & -1.34890 \\
\hline $\mathrm{H}$ & 4.13323 & -0.52202 & -1.88530 \\
\hline $\mathrm{C}$ & 1.73978 & -1.97060 & -1.46636 \\
\hline $\mathrm{H}$ & 1.33215 & -1.07667 & -1.94475 \\
\hline $\mathrm{H}$ & 0.98464 & -2.75726 & -1.49438 \\
\hline $\mathrm{C}$ & 3.07679 & -2.40173 & -2.06933 \\
\hline $\mathrm{H}$ & 3.08993 & -2.29482 & -3.15669 \\
\hline $\mathrm{H}$ & 3.27332 & -3.45110 & -1.82523 \\
\hline $\mathrm{N}$ & -6.04063 & 0.16656 & -0.45408 \\
\hline $\mathrm{O}$ & -6.38050 & 0.37966 & -1.62125 \\
\hline $\mathrm{O}$ & -6.84081 & -0.02850 & 0.46510 \\
\hline $\mathrm{C}$ & 1.77961 & -1.93789 & 2.38207 \\
\hline $\mathrm{H}$ & 1.79772 & -0.94271 & 2.84325 \\
\hline $\mathrm{H}$ & 2.78670 & -2.35690 & 2.44087 \\
\hline $\mathrm{H}$ & 1.11459 & -2.55562 & 2.98905 \\
\hline $\mathrm{C}$ & 1.25534 & -1.88656 & 0.97051 \\
\hline $\mathrm{C}$ & -0.14003 & -1.87546 & 0.76684 \\
\hline $\mathrm{H}$ & -0.74773 & -2.26337 & 1.57875 \\
\hline $\mathrm{H}$ & -0.53195 & -2.08615 & -0.22156 \\
\hline $\mathrm{C}$ & 3.79268 & 0.04469 & 0.73553 \\
\hline $\mathrm{H}$ & 3.63436 & -0.03367 & 1.81188 \\
\hline $\mathrm{H}$ & 4.83905 & 0.32343 & 0.58143 \\
\hline $\mathrm{C}$ & 2.92701 & 2.33150 & 1.26931 \\
\hline $\mathrm{H}$ & 3.96408 & 2.54086 & 1.54771 \\
\hline $\mathrm{H}$ & 2.36617 & 2.02892 & 2.15521 \\
\hline $\mathrm{C}$ & 3.26391 & 1.81444 & -1.06710 \\
\hline $\mathrm{H}$ & 2.64690 & 1.32608 & -1.82114 \\
\hline $\mathrm{H}$ & 4.31584 & 1.62593 & -1.28391 \\
\hline $\mathrm{C}$ & 2.30355 & 3.46705 & 0.46905 \\
\hline
\end{tabular}




\begin{tabular}{|c|c|c|c|}
\hline H & 2.49264 & 4.43943 & 0.92926 \\
\hline $\mathrm{H}$ & 1.22155 & 3.31665 & 0.40397 \\
\hline 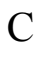 & 2.95980 & 3.32238 & -0.91827 \\
\hline I & 3.89249 & 3.89143 & -0.96111 \\
\hline $\mathrm{H}$ & 2.31274 & 3.68036 & -1.72181 \\
\hline $\mathrm{H}$ & 1.89918 & 0.86784 & 0.21099 \\
\hline \multicolumn{4}{|c|}{ Electronic energy $=-1130.539312$} \\
\hline \multirow{6}{*}{\multicolumn{4}{|c|}{$\begin{array}{l}\text { Zero-point electronic energy }=-1130.07755 \\
\text { Enthalpy }=-1130.053668 \\
\text { Free energy }=-1130.131654 \\
\text { Free energy with quasiharmonic approximat } \\
\text { Frequencies }=-269.524818 .405429 .4408-2 \\
\text { SCF (M06-2X/def2-TZVPP) }=-1130.48523\end{array}$}} \\
\hline & & & \\
\hline & & & \\
\hline & & & \\
\hline & & & \\
\hline & & & \\
\hline \multicolumn{4}{|c|}{ TS-2d } \\
\hline $\mathrm{C}$ & 3.65895 & -0.97279 & 0.45003 \\
\hline $\mathrm{H}$ & 3.89055 & -0.58781 & 1.44241 \\
\hline $\mathrm{N}$ & 2.33391 & -1.63249 & 0.44220 \\
\hline $\mathrm{N}$ & 2.81294 & 1.32495 & -0.37707 \\
\hline C & -2.66263 & -1.06188 & -0.94220 \\
\hline $\mathrm{C}$ & -1.90349 & -0.12884 & -0.21937 \\
\hline $\mathrm{C}$ & -2.55864 & 0.84636 & 0.54593 \\
\hline $\mathrm{C}$ & -3.94680 & 0.88725 & 0.60445 \\
\hline $\mathrm{C}$ & -4.67628 & -0.05782 & -0.11994 \\
\hline $\mathrm{C}$ & -4.05166 & -1.03425 & -0.89907 \\
\hline $\mathrm{H}$ & -1.96176 & 1.57641 & 1.08090 \\
\hline $\mathrm{H}$ & -4.46587 & 1.63466 & 1.19145 \\
\hline $\mathrm{H}$ & -4.64891 & -1.74561 & -1.45533 \\
\hline $\mathrm{C}$ & -0.39399 & -0.14514 & -0.27757 \\
\hline $\mathrm{O}$ & 0.22143 & 0.94310 & 0.08424 \\
\hline $\mathrm{H}$ & -2.16361 & -1.81274 & -1.54929 \\
\hline $\mathrm{H}$ & -0.03021 & -0.63390 & -1.19806 \\
\hline $\mathrm{C}$ & 4.59692 & -2.12346 & 0.04833 \\
\hline $\mathrm{H}$ & 4.80373 & -2.72949 & 0.93522 \\
\hline $\mathrm{H}$ & 5.55121 & -1.76704 & -0.34713 \\
\hline $\mathrm{C}$ & 2.30509 & -2.71792 & -0.58068 \\
\hline $\mathrm{H}$ & 1.67874 & -2.43492 & -1.43024 \\
\hline $\mathrm{H}$ & 1.86919 & -3.60474 & -0.11432 \\
\hline $\mathrm{C}$ & 3.78003 & -2.92559 & -0.98511 \\
\hline $\mathrm{H}$ & 3.95513 & -2.55182 & -1.99765 \\
\hline $\mathrm{H}$ & 4.04586 & -3.98467 & -0.97967 \\
\hline $\mathrm{N}$ & -6.13695 & -0.02042 & -0.06740 \\
\hline $\mathrm{O}$ & -6.76610 & -0.86102 & -0.71627 \\
\hline $\mathrm{O}$ & -6.67406 & 0.84893 & 0.62467 \\
\hline $\mathrm{C}$ & 1.42360 & -0.43324 & 2.39761 \\
\hline $\mathrm{H}$ & 1.02656 & 0.54356 & 2.07564 \\
\hline
\end{tabular}




$\begin{array}{lrrr}\mathrm{H} & 2.43592 & -0.32343 & 2.78577 \\ \mathrm{H} & 0.77690 & -0.79239 & 3.20208 \\ \mathrm{C} & 1.29681 & -1.34861 & 1.22419 \\ \mathrm{C} & -0.03283 & -1.67916 & 0.83543 \\ \mathrm{H} & -0.75831 & -1.65287 & 1.64337 \\ \mathrm{H} & -0.18670 & -2.50787 & 0.15070 \\ \mathrm{C} & 3.72106 & 0.15711 & -0.60234 \\ \mathrm{H} & 4.74314 & 0.54433 & -0.64027 \\ \mathrm{H} & 3.47585 & -0.24234 & -1.58887 \\ \mathrm{C} & 3.23024 & 2.26950 & 0.72342 \\ \mathrm{H} & 4.30658 & 2.42927 & 0.61758 \\ \mathrm{H} & 3.03273 & 1.81663 & 1.69416 \\ \mathrm{C} & 2.70680 & 2.21983 & -1.59190 \\ \mathrm{H} & 2.13410 & 1.69799 & -2.36001 \\ \mathrm{H} & 3.72193 & 2.39953 & -1.95661 \\ \mathrm{C} & 2.42522 & 3.55058 & 0.45265 \\ \mathrm{H} & 3.02669 & 4.42904 & 0.69618 \\ \mathrm{H} & 1.52755 & 3.57781 & 1.07426 \\ \mathrm{C} & 2.04238 & 3.49171 & -1.05447 \\ \mathrm{H} & 2.38477 & 4.36776 & -1.60926 \\ \mathrm{H} & 0.95848 & 3.41912 & -1.16517 \\ \mathrm{H} & 1.78573 & 1.02117 & -0.17940\end{array}$

Electronic energy $=-1130.536746$

Zero-point electronic energy $=-1130.075621$

Enthalpy $=-1130.051661$

Free energy $=-1130.129169$

Free energy with quasiharmonic approximation $=-1130.124727$

Frequencies $=-257.337728 .349532 .9336-257.337628 .15832 .8432$

SCF $($ M06-2X/def2-TZVPP $)=-1130.48338203$

TS-2e

$\begin{array}{lrrr}\mathrm{C} & -2.98840 & 1.33381 & 0.39459 \\ \mathrm{H} & -2.97221 & 0.98766 & 1.42842 \\ \mathrm{~N} & -1.61917 & 1.79396 & 0.01997 \\ \mathrm{~N} & -3.06037 & -1.14491 & -0.31174 \\ \mathrm{C} & 2.21281 & -0.40716 & -0.97416 \\ \mathrm{C} & 1.73670 & -0.75723 & 0.29766 \\ \mathrm{C} & 2.64701 & -0.91267 & 1.35510 \\ \mathrm{C} & 4.00651 & -0.70291 & 1.15895 \\ \mathrm{C} & 4.44920 & -0.34105 & -0.11568 \\ \mathrm{C} & 3.56999 & -0.19319 & -1.18936 \\ \mathrm{H} & 2.28994 & -1.20384 & 2.33942 \\ \mathrm{H} & 4.71886 & -0.81974 & 1.96580 \\ \mathrm{H} & 3.95153 & 0.07736 & -2.16593 \\ \mathrm{C} & 0.26857 & -1.02560 & 0.49635 \\ \mathrm{O} & -0.47308 & -1.12295 & -0.54497\end{array}$




\begin{tabular}{lrrr}
$\mathrm{H}$ & 1.50446 & -0.31996 & -1.79050 \\
$\mathrm{H}$ & 0.08711 & -1.70327 & 1.34806 \\
$\mathrm{C}$ & -3.86796 & 2.61982 & 0.25127 \\
$\mathrm{H}$ & -4.29459 & 2.89961 & 1.21681 \\
$\mathrm{H}$ & -4.70270 & 2.43272 & -0.43077 \\
$\mathrm{C}$ & -1.78532 & 2.91782 & -0.93052 \\
$\mathrm{H}$ & -2.05675 & 2.50754 & -1.90975 \\
$\mathrm{H}$ & -0.86525 & 3.48283 & -1.04837 \\
$\mathrm{C}$ & -2.93771 & 3.71273 & -0.31526 \\
$\mathrm{H}$ & -3.43702 & 4.34973 & -1.04931 \\
$\mathrm{H}$ & -2.55308 & 4.35111 & 0.48624 \\
$\mathrm{~N}$ & 5.87892 & -0.11864 & -0.33288 \\
$\mathrm{O}$ & 6.25410 & 0.19699 & -1.46515 \\
$\mathrm{O}$ & 6.64237 & -0.25556 & 0.62675 \\
$\mathrm{C}$ & 0.70284 & 2.44279 & 0.46339 \\
$\mathrm{H}$ & 1.03315 & 2.34875 & -0.57687 \\
$\mathrm{H}$ & 1.52772 & 2.14029 & 1.10790 \\
$\mathrm{H}$ & 0.48899 & 3.49991 & 0.65553 \\
$\mathrm{C}$ & -0.50667 & 1.58734 & 0.74120 \\
$\mathrm{C}$ & -0.36908 & 0.51589 & 1.64410 \\
$\mathrm{H}$ & 0.42031 & 0.61806 & 2.38121 \\
$\mathrm{H}$ & -1.26463 & 0.04124 & 2.03220 \\
$\mathrm{C}$ & -3.55659 & 0.24514 & -0.53492 \\
$\mathrm{H}$ & -4.64240 & 0.21692 & -0.41264 \\
$\mathrm{H}$ & -3.34294 & 0.49834 & -1.57685 \\
$\mathrm{C}$ & -3.51342 & -1.80008 & 0.98525 \\
$\mathrm{H}$ & -2.69632 & -1.72266 & 1.70360 \\
$\mathrm{H}$ & -4.37399 & -1.25188 & 1.37204 \\
$\mathrm{C}$ & -3.44832 & -2.08932 & -1.42654 \\
$\mathrm{H}$ & -2.80713 & -1.88804 & -2.28637 \\
$\mathrm{H}$ & -4.49161 & -1.89559 & -1.69297 \\
$\mathrm{C}$ & -3.85008 & -3.26192 & 0.62264 \\
$\mathrm{H}$ & -4.93524 & -3.39940 & 0.60668 \\
$\mathrm{H}$ & -3.43470 & -3.96059 & 1.35217 \\
$\mathrm{C}$ & -3.26727 & -3.46080 & -0.78885 \\
$\mathrm{H}$ & -2.20132 & -3.70846 & -0.74182 \\
-3.77795 & -4.24378 & -1.35405 \\
$\mathrm{H}$ & -1.97192 & -1.13617 & -0.33173 \\
& -1130.536963 \\
\hline
\end{tabular}

Zero-point electronic energy $=-1130.076545$

Enthalpy $=-1130.052243$

Free energy $=-1130.131967$

Free energy with quasiharmonic approximation $=-1130.125962$

Frequencies $=-294.243520 .116429 .4195-294.24217 .898428 .977$

SCF (M06-2X/def2-TZVPP) $=-1130.48253979$ 


\begin{tabular}{|c|c|c|c|}
\hline \multicolumn{4}{|c|}{ TS-2f } \\
\hline $\mathrm{C}$ & 3.64897 & -1.07194 & 0.70833 \\
\hline $\mathrm{H}$ & 3.94191 & -1.58376 & 1.63158 \\
\hline $\mathrm{N}$ & 2.34289 & -1.63236 & 0.25591 \\
\hline $\mathrm{N}$ & 2.79488 & 1.34549 & 0.21468 \\
\hline $\mathrm{C}$ & -2.66263 & -0.71349 & -1.18283 \\
\hline $\mathrm{C}$ & -1.91757 & -0.03572 & -0.20551 \\
\hline $\mathrm{C}$ & -2.58712 & 0.66593 & 0.80733 \\
\hline $\mathrm{C}$ & -3.97599 & 0.68265 & 0.85881 \\
\hline $\mathrm{C}$ & -4.69127 & -0.00698 & -0.12245 \\
\hline $\mathrm{C}$ & -4.05210 & -0.70486 & -1.14972 \\
\hline $\mathrm{H}$ & -2.00063 & 1.20508 & 1.54275 \\
\hline $\mathrm{H}$ & -4.50640 & 1.21962 & 1.63530 \\
\hline $\mathrm{H}$ & -4.63882 & -1.22026 & -1.89958 \\
\hline $\mathrm{C}$ & -0.40944 & -0.03064 & -0.24698 \\
\hline $\mathrm{O}$ & 0.19866 & 0.88804 & 0.44014 \\
\hline $\mathrm{H}$ & -2.15130 & -1.24780 & -1.97916 \\
\hline $\mathrm{H}$ & -0.02780 & -0.23029 & -1.26250 \\
\hline $\mathrm{C}$ & 4.63568 & -1.46831 & -0.41338 \\
\hline $\mathrm{H}$ & 5.61666 & -1.70398 & 0.00556 \\
\hline $\mathrm{H}$ & 4.77344 & -0.64803 & -1.12256 \\
\hline $\mathrm{C}$ & 2.48515 & -2.30842 & -1.06044 \\
\hline $\mathrm{H}$ & 2.20223 & -1.62777 & -1.87086 \\
\hline $\mathrm{H}$ & 1.83011 & -3.17989 & -1.09493 \\
\hline $\mathrm{C}$ & 3.97181 & -2.66311 & -1.10806 \\
\hline $\mathrm{H}$ & 4.32510 & -2.80914 & -2.13140 \\
\hline $\mathrm{H}$ & 4.15061 & -3.58638 & -0.54718 \\
\hline $\mathrm{N}$ & -6.15267 & 0.00721 & -0.07769 \\
\hline $\mathrm{O}$ & -6.76943 & -0.60374 & -0.95503 \\
\hline $\mathrm{O}$ & -6.70265 & 0.62812 & 0.83621 \\
\hline $\mathrm{C}$ & 1.20529 & -1.16085 & 2.37912 \\
\hline $\mathrm{H}$ & 0.88913 & -0.10495 & 2.34684 \\
\hline $\mathrm{H}$ & 0.44861 & -1.70640 & 2.94719 \\
\hline $\mathrm{H}$ & 2.16420 & -1.25006 & 2.89152 \\
\hline $\mathrm{C}$ & 1.21648 & -1.64666 & 0.96612 \\
\hline $\mathrm{C}$ & -0.05253 & -1.85195 & 0.35899 \\
\hline $\mathrm{H}$ & -0.84695 & -2.10190 & 1.05553 \\
\hline $\mathrm{H}$ & -0.10866 & -2.40658 & -0.57249 \\
\hline $\mathrm{C}$ & 3.63023 & 0.43580 & 1.06391 \\
\hline $\mathrm{H}$ & 3.26436 & 0.57623 & 2.08119 \\
\hline $\mathrm{H}$ & 4.66055 & 0.80250 & 1.03466 \\
\hline $\mathrm{C}$ & 2.74524 & 2.75099 & 0.77268 \\
\hline $\mathrm{H}$ & 2.19413 & 2.73487 & 1.71387 \\
\hline $\mathrm{H}$ & 3.77391 & 3.07378 & 0.95692 \\
\hline $\mathrm{C}$ & 3.21514 & 1.54316 & -1.22203 \\
\hline $\mathrm{H}$ & 2.91359 & 0.67758 & -1.81090 \\
\hline
\end{tabular}




$\begin{array}{llll}\mathrm{H} & 4.30466 & 1.62377 & -1.23628 \\ \mathrm{C} & 2.07079 & 3.54455 & -0.34546 \\ \mathrm{H} & 2.34497 & 4.60049 & -0.29937 \\ \mathrm{H} & 0.98597 & 3.46525 & -0.24272 \\ \mathrm{C} & 2.53883 & 2.85925 & -1.65825 \\ \mathrm{H} & 3.25454 & 3.47495 & -2.20770 \\ \mathrm{H} & 1.69275 & 2.66508 & -2.32106 \\ \mathrm{H} & 1.75121 & 1.02986 & 0.23384\end{array}$

Electronic energy $=-1130.534808$

Zero-point electronic energy $=-1130.073145$

Enthalpy $=-1130.049404$

Free energy $=-1130.12617$

Free energy with quasiharmonic approximation $=-1130.121812$

Frequencies $=-271.123630 .780632 .1410-271.123130 .679532 .0955$

SCF (M06-2X/def2-TZVPP) $=-1130.48168004$

TS-2g

$\begin{array}{lrrr}\mathrm{C} & 3.05507 & -1.31146 & 0.85784 \\ \mathrm{H} & 2.91451 & -1.85021 & 1.80112 \\ \mathrm{~N} & 1.89223 & -1.68832 & -0.01775 \\ \mathrm{~N} & 2.89801 & 1.23318 & 0.28192 \\ \mathrm{C} & -2.80839 & 0.35105 & 1.53302 \\ \mathrm{C} & -1.86324 & 0.48454 & 0.50342 \\ \mathrm{C} & -2.29871 & 0.50877 & -0.82913 \\ \mathrm{C} & -3.64875 & 0.38010 & -1.13766 \\ \mathrm{C} & -4.56308 & 0.23548 & -0.09344 \\ \mathrm{C} & -4.16149 & 0.22284 & 1.24453 \\ \mathrm{H} & -1.56377 & 0.64392 & -1.61461 \\ \mathrm{H} & -3.99813 & 0.39649 & -2.16246 \\ \mathrm{H} & -4.89958 & 0.11985 & 2.02984 \\ \mathrm{C} & -0.39817 & 0.67303 & 0.81390 \\ \mathrm{O} & 0.36285 & 1.04814 & -0.15873 \\ \mathrm{H} & -2.48292 & 0.35097 & 2.57007 \\ \mathrm{H} & -0.25650 & 1.14018 & 1.80389 \\ \mathrm{C} & 4.28613 & -1.89507 & 0.12440 \\ \mathrm{H} & 5.01378 & -2.27181 & 0.84727 \\ \mathrm{H} & 4.78615 & -1.12493 & -0.46937 \\ \mathrm{C} & 2.38828 & -2.38422 & -1.23700 \\ \mathrm{H} & 2.51860 & -1.65284 & -2.04239 \\ \mathrm{H} & 1.67349 & -3.13117 & -1.57317 \\ \mathrm{C} & 3.72170 & -2.98127 & -0.79390 \\ \mathrm{H} & 4.37411 & -3.20370 & -1.64162 \\ \mathrm{H} & 3.54605 & -3.90993 & -0.24043 \\ \mathrm{~N} & -5.98547 & 0.10180 & -0.40803 \\ \mathrm{O} & -6.77991 & -0.02587 & 0.52758 \\ \mathrm{O} & -6.32472 & 0.12124 & -1.59428\end{array}$




$\begin{array}{lrrr}\mathrm{C} & -0.38183 & -2.46651 & -0.47297 \\ \mathrm{H} & -0.15121 & -3.53407 & -0.56388 \\ \mathrm{H} & -0.39734 & -2.03812 & -1.48112 \\ \mathrm{H} & -1.37830 & -2.37135 & -0.04299 \\ \mathrm{C} & 0.62614 & -1.77052 & 0.40066 \\ \mathrm{C} & 0.16007 & -1.08738 & 1.54680 \\ \mathrm{H} & -0.75376 & -1.48260 & 1.97837 \\ \mathrm{H} & 0.88724 & -0.78654 & 2.29422 \\ \mathrm{C} & 3.23786 & 0.17099 & 1.27291 \\ \mathrm{H} & 2.63580 & 0.39738 & 2.15445 \\ \mathrm{H} & 4.28629 & 0.29558 & 1.56111 \\ \mathrm{C} & 3.06239 & 2.62817 & 0.86783 \\ \mathrm{H} & 2.12401 & 2.88211 & 1.36313 \\ \mathrm{H} & 3.86015 & 2.59853 & 1.61220 \\ \mathrm{C} & 3.62251 & 1.25482 & -1.03545 \\ \mathrm{H} & 3.26399 & 0.43909 & -1.66289 \\ \mathrm{H} & 4.69369 & 1.13529 & -0.84820 \\ \mathrm{C} & 3.40276 & 3.54588 & -0.32568 \\ \mathrm{H} & 4.42174 & 3.92800 & -0.21943 \\ \mathrm{H} & 2.72846 & 4.40340 & -0.37714 \\ \mathrm{C} & 3.29205 & 2.64445 & -1.57251 \\ \mathrm{H} & 3.97319 & 2.94000 & -2.37362 \\ \mathrm{H} & 2.27245 & 2.65238 & -1.97141 \\ \mathrm{H} & 1.82088 & 1.13745 & 0.07432 \\ \mathrm{Electronic} \text { energy } & -1130.532794 \\ \mathrm{Z} & -10101\end{array}$

Zero-point electronic energy $=-1130.072443$

Enthalpy $=-1130.048317$

Free energy $=-1130.127276$

Free energy with quasiharmonic approximation $=-1130.121562$

Frequencies $=-316.962618 .888027 .4607-316.962617 .99826 .8904$

SCF (M06-2X/def2-TZVPP) $=-1130.48086777$

TS-2h

$\begin{array}{lrrr}\mathrm{C} & 3.19905 & -0.73767 & 0.81644 \\ \mathrm{H} & 2.60960 & -0.36709 & 1.65583 \\ \mathrm{~N} & 2.45251 & -1.81787 & 0.12501 \\ \mathrm{~N} & 2.65701 & 1.56871 & -0.20503 \\ \mathrm{C} & -2.53315 & 1.00032 & 0.31096 \\ \mathrm{C} & -1.95816 & -0.01780 & -0.46355 \\ \mathrm{C} & -2.77583 & -1.02770 & -0.99255 \\ \mathrm{C} & -4.14398 & -1.03584 & -0.74548 \\ \mathrm{C} & -4.68636 & -0.01637 & 0.03919 \\ \mathrm{C} & -3.89792 & 1.00619 & 0.57168 \\ \mathrm{H} & -2.34190 & -1.80836 & -1.61162 \\ \mathrm{H} & -4.78701 & -1.80618 & -1.15190 \\ \mathrm{H} & -4.35595 & 1.78456 & 1.16901\end{array}$




\begin{tabular}{|c|c|c|c|}
\hline $\mathrm{C}$ & -0.48252 & 0.01057 & -0.76205 \\
\hline $\mathrm{O}$ & 0.13030 & 1.12772 & -0.66161 \\
\hline $\mathrm{H}$ & -1.89692 & 1.78990 & 0.69521 \\
\hline $\mathrm{H}$ & -0.20468 & -0.67829 & -1.57599 \\
\hline $\mathrm{C}$ & 4.45602 & -1.49005 & 1.29212 \\
\hline $\mathrm{H}$ & 5.26834 & -0.81598 & 1.57404 \\
\hline $\mathrm{H}$ & 4.19595 & -2.09626 & 2.16585 \\
\hline $\mathrm{C}$ & 3.40962 & -2.81469 & -0.43931 \\
\hline $\mathrm{H}$ & 3.35371 & -2.82206 & -1.53164 \\
\hline $\mathrm{H}$ & 3.13944 & -3.80898 & -0.07737 \\
\hline $\mathrm{C}$ & 4.79009 & -2.38752 & 0.09041 \\
\hline $\mathrm{H}$ & 5.34378 & -1.82770 & -0.66976 \\
\hline $\mathrm{H}$ & 5.39494 & -3.25376 & 0.36709 \\
\hline $\mathrm{N}$ & -6.12502 & -0.01453 & 0.30382 \\
\hline $\mathrm{O}$ & -6.58952 & 0.89666 & 0.99425 \\
\hline $\mathrm{O}$ & -6.80701 & -0.92492 & -0.17460 \\
\hline $\mathrm{C}$ & 0.62632 & -3.32653 & -0.41990 \\
\hline $\mathrm{H}$ & 0.99535 & -4.20702 & 0.11948 \\
\hline $\mathrm{H}$ & -0.46351 & -3.35051 & -0.39458 \\
\hline $\mathrm{H}$ & 0.95462 & -3.41805 & -1.46068 \\
\hline $\mathrm{C}$ & 1.13611 & -2.05748 & 0.21604 \\
\hline $\mathrm{C}$ & 0.19018 & -1.17657 & 0.77763 \\
\hline $\mathrm{H}$ & -0.72011 & -1.66007 & 1.11449 \\
\hline $\mathrm{H}$ & 0.51780 & -0.36651 & 1.42047 \\
\hline $\mathrm{C}$ & 3.60224 & 0.41581 & -0.13964 \\
\hline $\mathrm{H}$ & 4.56647 & 0.82495 & 0.17303 \\
\hline $\mathrm{H}$ & 3.71008 & 0.03885 & -1.15880 \\
\hline $\mathrm{C}$ & 3.00091 & 2.54524 & -1.32346 \\
\hline $\mathrm{H}$ & 4.06824 & 2.46059 & -1.53364 \\
\hline $\mathrm{H}$ & 2.43847 & 2.22859 & -2.20299 \\
\hline $\mathrm{C}$ & 2.54475 & 2.39744 & 1.05055 \\
\hline $\mathrm{H}$ & 1.95002 & 1.86070 & 1.79026 \\
\hline $\mathrm{H}$ & 3.55365 & 2.55808 & 1.44141 \\
\hline $\mathrm{C}$ & 2.60982 & 3.94285 & -0.79870 \\
\hline $\mathrm{H}$ & 1.96583 & 4.46980 & -1.50608 \\
\hline $\mathrm{H}$ & 3.50940 & 4.54693 & -0.65210 \\
\hline $\mathrm{C}$ & 1.90389 & 3.68758 & 0.54949 \\
\hline $\mathrm{H}$ & 2.03703 & 4.50570 & 1.26092 \\
\hline $\mathrm{H}$ & 0.83192 & 3.52670 & 0.40219 \\
\hline $\mathrm{H}$ & 1.65739 & 1.23484 & -0.43216 \\
\hline
\end{tabular}

Electronic energy $=-1130.535839$

Zero-point electronic energy $=-1130.075156$

Enthalpy $=-1130.050756$

Free energy $=-1130.130473$

Free energy with quasiharmonic approximation $=-1130.124686$

Frequencies $=-292.178719 .019327 .9126-292.178418 .134227 .8215$ 
SCF $($ M06-2X/def2-TZVPP $)=-1130.47796747$

TS-2h

\begin{tabular}{|c|c|c|c|}
\hline $\mathrm{C}$ & -3.30384 & -0.99861 & -0.53215 \\
\hline $\mathrm{H}$ & -2.95437 & -0.67816 & -1.52258 \\
\hline $\mathrm{N}$ & -2.07836 & -1.63475 & 0.02575 \\
\hline $\mathrm{C}$ & -1.45520 & -1.47178 & 1.17284 \\
\hline $\mathrm{C}$ & -0.00912 & -1.55849 & 1.17555 \\
\hline $\mathrm{H}$ & 0.42411 & -2.13593 & 0.36135 \\
\hline $\mathrm{H}$ & 0.43799 & -1.81295 & 2.13545 \\
\hline $\mathrm{C}$ & -3.81970 & 0.27247 & 0.12832 \\
\hline $\mathrm{H}$ & -4.04851 & 0.15992 & 1.18548 \\
\hline $\mathrm{C}$ & -2.82048 & 2.04711 & -1.38852 \\
\hline $\mathrm{C}$ & -3.06498 & 2.53413 & 0.92975 \\
\hline $\mathrm{H}$ & -2.38082 & 1.36789 & -2.11979 \\
\hline $\mathrm{H}$ & -3.85423 & 2.27178 & -1.67095 \\
\hline $\mathrm{C}$ & -1.99427 & 3.30813 & -1.14571 \\
\hline $\mathrm{C}$ & -2.41269 & 3.77076 & 0.27265 \\
\hline $\mathrm{H}$ & -2.64054 & 2.27185 & 1.90037 \\
\hline $\mathrm{H}$ & -4.14522 & 2.65710 & 1.04530 \\
\hline $\mathrm{H}$ & -0.93315 & 3.04688 & -1.16395 \\
\hline $\mathrm{H}$ & -2.17998 & 4.06367 & -1.91226 \\
\hline $\mathrm{H}$ & -1.55220 & 4.12047 & 0.84739 \\
\hline $\mathrm{N}$ & -2.82957 & 1.39379 & -0.02988 \\
\hline $\mathrm{C}$ & 2.80652 & -0.01875 & 1.51063 \\
\hline $\mathrm{C}$ & 1.84670 & 0.07119 & 0.49165 \\
\hline $\mathrm{C}$ & 2.26661 & 0.14169 & -0.84328 \\
\hline $\mathrm{C}$ & 3.61919 & 0.11641 & -1.16443 \\
\hline $\mathrm{C}$ & 4.55208 & 0.02188 & -0.12974 \\
\hline $\mathrm{C}$ & 4.16408 & -0.04390 & 1.20989 \\
\hline $\mathrm{H}$ & 1.51501 & 0.23078 & -1.61943 \\
\hline $\mathrm{H}$ & 3.95736 & 0.17223 & -2.19168 \\
\hline $\mathrm{H}$ & 4.91429 & -0.10781 & 1.98795 \\
\hline $\mathrm{C}$ & 0.35953 & 0.09968 & 0.81649 \\
\hline $\mathrm{O}$ & -0.40575 & 0.59517 & -0.15818 \\
\hline $\mathrm{H}$ & 2.49133 & -0.06068 & 2.55000 \\
\hline $\mathrm{H}$ & 0.22439 & 0.56843 & 1.81006 \\
\hline $\mathrm{H}$ & -3.13585 & 4.58887 & 0.22855 \\
\hline $\mathrm{H}$ & -4.74736 & 0.56311 & -0.37469 \\
\hline $\mathrm{C}$ & -4.20449 & -2.20896 & -0.79054 \\
\hline $\mathrm{H}$ & -4.98140 & -1.98414 & -1.52464 \\
\hline $\mathrm{H}$ & -4.68675 & -2.54311 & 0.13299 \\
\hline $\mathrm{C}$ & -1.76094 & -2.69504 & -0.96474 \\
\hline $\mathrm{H}$ & -1.10099 & -3.43637 & -0.51828 \\
\hline $\mathrm{H}$ & -1.26932 & -2.24996 & -1.83356 \\
\hline $\mathrm{C}$ & -3.17461 & -3.25994 & -1.31020 \\
\hline
\end{tabular}




$\begin{array}{lrrr}\mathrm{H} & -3.32613 & -4.22805 & -0.82849 \\ \mathrm{H} & -3.26326 & -3.40440 & -2.38926 \\ \mathrm{~N} & 5.97686 & -0.00258 & -0.45722 \\ \mathrm{O} & 6.78766 & -0.08817 & 0.46948 \\ \mathrm{O} & 6.30417 & 0.06173 & -1.64553 \\ \mathrm{C} & -2.12398 & -0.93913 & 2.40776 \\ \mathrm{H} & -3.17362 & -1.22558 & 2.46936 \\ \mathrm{H} & -1.60449 & -1.35394 & 3.27556 \\ \mathrm{H} & -2.04008 & 0.15052 & 2.49290 \\ \mathrm{H} & -1.73985 & 1.00957 & 0.07276 \\ \text { Electronic energy }=-1130.516476 & \\ \text { Zero-point electronic energy }=-1130.057547 \\ \text { Enthalpy = -1130.033553 } \\ \text { Free energy = -1130.111251 } \\ \text { Free energy with quasiharmonic approximation = }-1130.106555 \\ \text { Frequencies = -236.0355 29.1794 32.8097 -236.0280 29.068932.7198 } \\ \text { SCF (M06-2X/def2-TZVPP) =-1130.468315 }\end{array}$

